\title{
Enhancing Image Denoising Performance of Bidimensional Empirical Mode Decomposition by Improving the Edge Effect
}

\author{
Feng-Ping An, ${ }^{1}$ Da-Chao Lin, ${ }^{2}$ Xian-Wei Zhou, ${ }^{1}$ and Zhihui Sun ${ }^{3}$ \\ ${ }^{1}$ School of Computer and Communication Engineering, University of Science and Technology Beijing, Beijing 100083, China \\ ${ }^{2}$ Department of Civil Engineering, North China Institute of Science and Technology, Yanjiao, Beijing 101601, China \\ ${ }^{3}$ School of Government, Central University of Finance and Economics, Beijing 100081, China \\ Correspondence should be addressed to Feng-Ping An; anfengping1985@163.com
}

Received 20 April 2015; Accepted 31 August 2015

Academic Editor: Atsushi Mase

Copyright (C) 2015 Feng-Ping An et al. This is an open access article distributed under the Creative Commons Attribution License, which permits unrestricted use, distribution, and reproduction in any medium, provided the original work is properly cited.

\begin{abstract}
Bidimensional empirical mode decomposition (BEMD) algorithm, with high adaptive ability, provides a suitable tool for the noisy image processing, and, however, the edge effect involved in its operation gives rise to a problem-how to obtain reliable decomposition results to effectively remove noises from the image. Accordingly, we propose an approach to deal with the edge effect caused by BEMD in the decomposition of an image signal and then to enhance its denoising performance. This approach includes two steps, in which the first one is an extrapolation operation through the regression model constructed by the support vector machine (SVM) method with high generalization ability, based on the information of the original signal, and the second is an expansion by the closed-end mirror expansion technique with respect to the extrema nearest to and beyond the edge of the data resulting from the first operation. Applications to remove the Gaussian white noise, salt and pepper noise, and random noise from the noisy images show that the edge effect of the BEMD can be improved effectively by the proposed approach to meet requirement of the reliable decomposition results. They also illustrate a good denoising effect of the BEMD by improving the edge effect on the basis of the proposed approach. Additionally, the denoised image preserves information details sufficiently and also enlarges the peak signal-to-noise ratio.
\end{abstract}

\section{Introduction}

In these years, digital image processing technology has been widely used in space exploration, remote sensing, biomedical and industrial inspection, and various other fields. Although it has made a great progress in many aspects, there are still some new challenges. One of them is closely related to image denoising, since noises can be introduced by almost all of the factors in the process of signal acquisition, conversion, and transfer, not to mention those involved in the original image from the instruments themselves, and environments. In order to obtain real information for a piece of image, denoising generally constitutes a necessary task before the implementation of the postanalysis (such as compression, split, and merge), aiming at improving the signal-to-noise ratio (SNR) and restoring true information of the image.

In the process of image denoising, it is necessary to eliminate the noise contaminating a given image as far as possible and also to preserve all important details of the image. To meet these requirements, a number of theoretical methods have been proposed and developed [1-5]. Relatively, techniques based on Fourier transform and wavelet transform have received more attention than the others in applications to image denoising [3,6-8]. At the same time, it is also found that they have some limitations. The methods based on Fourier transform can run well for parts of the texture with some deterministic characteristics or of the change with gentle slopes, while they cannot give a satisfactory performance for those parts of the texture with sharp changes in the image. Although wavelet transform provides a useful description in time-frequency domain for some sudden changes of the image contents or slow ones of the texture, a complete description for both of them is still a hard work.

With the development of artificial intelligence algorithms, there have appeared a number of new attempts in 
image denoising. From a new view, the application of artificial neural network (ANN) fully shows advantages of parallel computing, nonlinear mapping, and adaptive capacity in image processing [9-13]. A good denoising effect especially can be presented by the method of pulse coupled ANN $[9,10$, 12]. Another representative technique is the so-called support vector machine (SVM) method. Based on statistical learning theory and structural risk minimization principle, with a high degree of generalization ability, fascinating learning efficiency, and other characteristics [14], it has attracted attention of the denoising researches. Unfortunately, almost all of these methods based on artificial intelligence algorithms require a requisite of signal preprocessing so as to obtain optimal model assumptions and simultaneously to carry out the model training processes. Consequently, there are some difficulties to make full use of their adaptive ability, which limit their extensive applications in image denoising.

For the adaptive ability of image denoising, one of its most important senses addresses the idea that its denoised version can preserve the image features as far as possible, with little influence on the following analysis and processing of the image. However, such influence, even the image distortion, can be found after the actual denoising operation. It is just a view of the limitation of the techniques based on traditional Fourier transform, wavelet transform, and modern artificial intelligence algorithms. To be worthy of note, Huang et al. [15] proposed a feasible technical approach, referred to as empirical mode decomposition (EMD) method, able to solve this problem to some extent. The EMD is a technique suitable for nonstationary time-frequency analysis and has been applied in earthquake [16], structural diagnosis [17], biological [18], mechanical fault diagnosis [19, 20], marine [21], and other fields. Compared with traditional Fourier transform and wavelet transform, it abandons the requirement of choosing a priori basis function, completely relying on the data feature itself through data-driven implementation of the decomposition process. Thus, the local variation characteristics may be seen clearly in time-frequency domain from the signal by using this method.

About ten years ago, Nunes et al. [22, 23] introduced the EMD into image processing and proposed the algorithm of bidimensional empirical mode decomposition (BEMD). Soon afterward, this algorithm received attention from some researchers and was put into image compression $[24,25]$, image texture classification [26], image denoising [27], image texture segmentation [28, 29], image scaling [30], texture synthesis [31], and image feature extraction [32]. Due to the noise characteristics of practical images, it is generally likely that the highest noise content of an image is extracted into the first mode component for the natural property of the BEMD process, and the remaining fewer noise contents sneak into the subsequent ones. On the basis of this fact, the image denoising algorithm by using the BEMD is devised $[23,27]$. It is known that the BEMD has a close relation with correct interpolation operation. But, at the boundary region, incorrect interpolation may occur for lack of constraints from extrema points. This is the so-called edge effect and also an open problem in the BEMD. However, it has not been solved so well that further works are required urgently in the application of the BEMD.

In fact, for the decomposition of a one-dimensional signal by the EMD, the edge effects or end effects may be deleted more or less by some strategies such as mirror expansion [33], neural networks expansion [34,35], ARMA model prediction of time series [36, 37], polynomial fitting extrapolation [38], and waveform feature match expansion [39]. Once these end effects are mitigated, more satisfactory decomposed results can be obtained, which shows that treatment of the end effect is beneficial for the application of the EMD. Accordingly, improvement of the edge effects for the image signals also should be significant to the development of the image denoising by using the BEMD.

To this end, we propose an approach of improving the edge effect of the BEMD to enhance its image denoising performance. This approach applies the SVM method [40, 41] to construct a regression model of the digital image. By using this model, an extrapolation operation is done to have a guess of the image beyond its boundaries. The extrema of the extrapolation signals neighboring the edges are taken as positions of the mirror, and then the closedend mirroring expansion is introduced to further remove the edge effects of the BEMD. Some case studies of removing the Gaussian white noise, salt and pepper noise, and random noise from the noisy images illustrate a good denoising result of the BEMD coupled with the proposed approach, and the denoised version of the image can also maintain its original details to the full extent.

\section{Fundamentals of BEMD Algorithm}

Similar to the EMD of the one-dimensional case, the BEMD uses the extrema that are found in the original image or obtained from the first derivative of the original or the higher-order derivative, to achieve the decomposition of the image signal. Distances between extrema may provide the information for characterizing the image on intrinsic length scales.

For a two-dimensional image denoted by $f(m, n)$, the basic procedure of the BEMD may be summarized as follows $[15,18,22,23]$ :

(1) Initialize the image under consideration, $r_{0}(m, n)=$ $f(m, n), k=1,(m, n) \in[0, M-1] \times[0, N-1]$, where $M$ and $N$ represent numbers of the rank on the discrete image plane.

(2) Initialize the parameters, $h_{k, 0}(m, n)=r_{k-l}(m, n)$, and $l=1$.

(3) Extract extrema points of $h_{k, l-1}(m, n)$.

(4) By cubic spline, interpolate between local maxima and between minima, respectively, to get two envelope surfaces $e_{\max , l-1}(m, n)$ and $e_{\min , l-1}(m, n)$ of $h_{k, l-1}(m, n)$.

(5) Calculate the mean envelope surface in terms of these two envelope surfaces, given by

$e_{\text {mean }, l-1}(m, n)=\frac{1}{2}\left[e_{\max , l-1}(m, n)+e_{\min , l-1}(m, n)\right]$. 
(6) Update the original signal and designate a new one for iteration, given by

$$
\begin{aligned}
h_{k, l}(m, n)=k_{k, l-1}(m, n)-e_{\text {mean }, l-1}(m, n), & \\
& l \Longrightarrow l+1 .
\end{aligned}
$$

(7) Calculate the standard deviation, SD, given by

$$
\mathrm{SD}=\sum_{m=0}^{M} \sum_{n=0}^{N} \frac{\left|h_{k, l-1}(m, n)-h_{k, l}(m, n)\right|^{2}}{h_{k, l-1}^{2}(m, n)}
$$

(8) Repeat steps (2) to (7) until the calculated standard deviation is less than a predetermined criterion (SD) (generally taken as $0.2-0.3$ ) and stop the iteration. Right now, it is regarded that $h_{k, l}(m, n)$ represents an intrinsic mode function (IMF); that is, $\operatorname{imf}_{k}(m, n)=$ $h_{k, l}(m, n)$.

(9) Update this signal and obtain the residual signal

$$
r_{k}(m, n)=r_{k-1}(m, n)-\operatorname{imf}_{k}(m, n) .
$$

(10) Repeat steps (1) to (9) over and over again, up to the $K$ th times, $K=(k+1)$; when the residual $r_{K}(m, n)$ is a monotonic signal, stop the process of BEMD and finally obtain all IMF components.

Once the decomposition process has finished, the original image can be expressed as the sum of all IMFs and the residual $r_{K}$, given by

$$
f(m, n)=\sum_{k=1}^{K} \operatorname{imf}_{k}(m, n)+r_{K}(m, n) .
$$

Stopping criterion directly decides the end of the decomposition process and also determines the corresponding final residual term. Therefore, the choice of the value of SD has a close relation with the number of the IMFs obtained by the BEMD. It is necessary to properly choose the value of SD on the basis of the actual situation. In order to portray details of the image, it should be a best choice of a lower value of the $\mathrm{SD}$, generally taken as $0.2-0.3$.

In decomposing a one-dimensional signal through the EMD, the residual presents a trend varying with time, which can be ignored in the following analyses. However, the residual resulting from the decomposition of a twodimensional signal through the BEMD often contains some information of the original image characteristics. It cannot be ignored in the following works, and its influence must be considered for some contributions to the original image.

\section{Treatment of the Edge Effect}

It is an open problem that there is a certain edge effect in the process of the BEMD. Generally, an actual image signal is not so long or even very short, and serious edge effects can be seen sometimes for its decomposition. Such decomposition is a process screening one after another for many times. With the progression of this process, the edge effect will be more serious, leading to distortions of the IMFs of the image signal decomposed by the BEMD. To this end, some treatments should be taken to mitigate or eliminate edge effects occurring in the screening process of the BEMD operation. Combining advantages of the SVM method with those of the mirror expansion, a new approach to deal with the edge effect caused by BEMD in the decomposition of an image signal is proposed as follows.

\subsection{SVM Regression Model and Its Extrapolation}

3.1.1. Regression Model. Given training sampling data $X=$ $\left\{\left(x_{1}, y_{1}\right), \ldots,\left(x_{i}, y_{i}\right)\right\}$, where $x_{i}$ is an input vector of $m$ columns, $x_{i} \in R^{m}$, and $y_{i}$ is the output value corresponding to $x_{i}, y_{i} \in R$, may be transformed into a high dimensional feature space $F$ by the SVM regression through a nonlinear mapping $\phi$. In this feature space, one can operate a linear regression to get its regression function, expressed as

$$
y=f(x)=\{w, \phi(x)\}+b
$$

In this equation, $\{$,$\} represents the inner product, w$ is the complexity of the function $f(x)$ described by the original data and transforming them into a high-dimensional space $R^{m}$, and $b$ is a constant, as the bias term, $b \in R$. Equation (6) requires constraint conditions:

$$
\begin{aligned}
y_{i}-w^{T} \cdot \phi\left(x_{i}\right)-b & \leq \varepsilon+\xi_{i} \quad(i=1,2, \ldots, l), \\
w^{T} \cdot \phi\left(x_{i}\right)+b & \leq \varepsilon+\xi_{i}^{*}, \\
\xi_{i}^{*}, \xi_{i} & \geq 0, \quad(i=1,2, \ldots, l) .
\end{aligned}
$$

The objective function is

$$
\min _{\omega, b, \xi_{i}, \xi_{i}^{*}} \frac{1}{2} \omega \omega^{T}+C \sum_{i=1}^{l}\left(\xi_{i}+\xi_{i}^{*}\right)
$$

where $\xi_{i}$ and $\xi_{i}^{*}$ are the slack variables, denoting the upper and lower errors of the training $y_{i}-w^{T} \cdot \phi\left(x_{i}\right)-b$ under the constraint of the error $\varepsilon$ and $C$ is a constant to control the penalty degree for the sampling data out of $\varepsilon, C>0$. Vapnik proposed that the risk is measured by the $\varepsilon$-insensitive loss function as follows [40]:

$$
|f(x)-y|= \begin{cases}|f(x)-y|-\varepsilon & (|f(x)-y| \geq \varepsilon), \\ 0 & (|f(x)-y|<\varepsilon) .\end{cases}
$$

When the absolute value of the difference between the actual and predicted values is less than $\varepsilon$, the difference for both of them will be negligible, or otherwise it should be equal to $|f(x)-y|-\varepsilon$. 
The optimization problem presented by (8) may be solved by means of Lagrangian penalty method, in which the Lagrangian function is expressed as [40]

$$
\begin{aligned}
L= & \frac{1}{2} \omega^{T}-\omega+C \sum_{i=1}^{l}\left(\xi_{i}+\xi_{i}^{*}\right) \\
& -\sum_{i=1}^{l} \alpha_{i}\left(\xi_{i}+\varepsilon-y_{i}+\omega x_{i}+b\right) \\
& -\sum_{i=1}^{l} \alpha_{i}^{*}\left(\xi_{i}+\varepsilon+y_{i}-\omega x_{i}-b\right) \\
& -\sum_{i=1}^{l}\left(\eta_{i} \xi_{i}-\eta_{i}^{*} \xi_{i}^{*}\right),
\end{aligned}
$$

where $\alpha_{i}, \alpha_{i}^{*}, \eta_{i}$, and $\eta_{i}^{*}$ are Lagrange multipliers and all of them are greater than zero. According to the KKT conditions, one has [40]

$$
\begin{aligned}
& \partial_{b} L=0 \longrightarrow \sum_{i=1}^{l}\left(\alpha_{i}-\alpha_{i}^{*}\right)=0, \\
& \alpha_{i} \geq 0, \\
& \alpha_{i}^{*} \leq C, \\
& \partial_{\omega} L=0 \longrightarrow \omega=\sum_{i=1}^{l}\left(\alpha_{i}-\alpha_{i}^{*}\right) \phi\left(x_{i}\right), \\
& \quad(i=1,2, \ldots, l) .
\end{aligned}
$$

At last, the nonlinear regression problem is transformed into a dual problem of (7), given by

$$
\begin{aligned}
\min _{\alpha_{i}, \alpha_{i}^{*}} W\left(\alpha_{i}, \alpha_{i}^{*}\right) & \\
= & -\frac{1}{2} \sum_{j=1}^{l}\left(\alpha_{i}+\alpha_{i}^{*}\right)\left(\alpha_{j}+\alpha_{j}^{*}\right) K\left(x_{i}, x_{j}\right) \\
& -\varepsilon \sum_{j=1}^{l}\left(\alpha_{i}+\alpha_{i}^{*}\right)+\sum_{j=1}^{l} y_{i}\left(\alpha_{i}-\alpha_{i}^{*}\right),
\end{aligned}
$$

where $K\left(x_{i}, x_{j}\right)(i, j=1,2, \ldots, l)$ is the kernel function of the SVM. After solving this optimization problem, one can determine parameters $\alpha_{i}$ and $\alpha_{i}^{*}$. After that, substituting them into (11) yields the other parameters of the SVM and finally gets the regression model as follows:

$$
y=f(x)=\sum_{i=1}^{l}\left(\alpha_{i}-\alpha_{i}^{*}\right) K\left(x, x_{i}\right)+b
$$

Since the regression model is driven by and based on characteristics of the training data, an obvious adaptive ability can be effectively guaranteed in the regression process if appropriate input nodes and related parameters are chosen. Therefore, the SVM regression modeling may provide a good foundation for the expansion of the image data in the following operation.
3.1.2. Parameter Optimization. In order for support vector machine (SVM) algorithm to have a better effect, this paper uses the particle swarm algorithm to improve support vector machine (SVM) parameter, such as $\xi_{i}, \xi_{i}^{*}$, and $C$. The basic principle is as follows.

The algorithm divides particles into two parts according to the fitness value; the fitness value is the standard of measuring the particle's quality. It distinguishes the particle's quality of the wheat from the chaff through comparing a single particle fitness value and the group average fitness value and then adjusts reasonably the particle's search ability $\omega$ and learning abilities $c_{1}, c_{2}$. It is advantageous to the particle swarm fast convergence and improves the accuracy of particle swarm search. $\omega$ is randomness; it can make $c_{1}, c_{2}$ avoid convergence of all particles in a certain direction and reduce the likelihood of being trapped in local optimum. In the end, it gets the optimal parameters of the support vector machine (SVM) model. The calculation method of $\omega, c_{1}$, and $c_{2}$ is as follows:

$$
\begin{aligned}
& \omega=\omega_{0}+k_{1} * \mu * x *(1-x), \\
& c_{1}=c_{10}-k_{2} * \mu * x *(1-x), \\
& c_{2}=c_{20}+k_{3} * \mu * x *(1-x), \\
& \omega=\omega_{0}-k_{1} * \mu * x *(1-x), \\
& c_{1}=c_{10}+k_{2} * \mu * x *(1-x), \\
& c_{2}=c_{20}-k_{3} * \mu * x *(1-x),
\end{aligned}
$$

$$
f<f_{\text {avg }} .
$$

In the above formula, $0<\omega_{0}<1,1<c_{10}<2,1<c_{20}<2$, $k_{1}, k_{2}$, and $k_{3}$ are constant numbers from 0 to $1, \mu=4, x$ is a random number from 0 to $1, f$ is the $i$ th particle fitness value of the iter generation, and $f_{\text {avg }}$ is the average fitness of all particles of the iter generation.

When $f>f_{\text {avg }}$, the particle's quality is poor and (14) will reduce the self-learning ability $c_{1}$ by enhancing the global search ability $\omega$ and the social learning ability $c_{2}$. It will make the particles "fly" quickly toward the direction of the optimal solution and improve the quality of particles. When $f<f_{\text {avg }}$, the particle's quality is better and (15) will reduce social learning ability $c_{2}$ by enhancing the particle's local search ability $\omega$ and self-learning ability $c_{1}$. The algorithm is conducive to finding the global optimal solution of support vector machine (SVM) algorithm parameters and reducing the likelihood of being trapped in local optimum.

3.1.3. Extrapolation Expansion. For a two-dimensional image $f(m, n)$, it can be described by a certain type of digital signal and forms a data matrix. In the theoretical frame of the SVM modeling, the data can be used to construct the training sampling data and to get the corresponding regression model. After that, the regression model is extrapolated towards outside regions of the image to realize its extrapolation expansion whose procedure may be summarized as follows. 
(1) For all of the sampling data in the matrix, indexes of the column are regarded as discrete variable $x_{i}$ in the SVM regression model, and elements of the matrix are output values of $f(x)$ for the model.

(2) Choosing these data $X=\left\{\left(x_{1}, y_{1}\right), \ldots,\left(x_{i}, y_{i}\right)\right\}$ as a sequence of the sampling, one can calculate the related parameters in the SVM modeling and then construct the corresponding SVM regression model, where the radial basis kernel (RBF) is used as the kernel function and the penalty coefficient $C$ takes a value of 10 .

(3) The regression model trained in step (2) extrapolates $m$ data values $\left\{\left(x_{i+1}, y_{i+1}\right), \ldots,\left(x_{i+m}, y_{i+m}\right)\right\}$ toward the outside regions on the left and right sides of the image, respectively, where $x_{i+m}$ represents the index of the expanded column and $y_{i+m}$ is the forecasting value of extrapolation at the position $x_{i+m}$.

(4) Further, indexes of the row are regarded as the variable $x_{i}$ of SVM regression model, and elements of the matrix are values of $f(x)$ corresponding to different $x_{i}$.

(5) The sampling data $X=\left\{\left(x_{1}, y_{1}\right), \ldots,\left(x_{i}, y_{i}\right)\right\}$ may be given and, similar to step (2), applied to construct SVM regression model.

(6) The obtained regression model trained from step (5) extrapolates $m$ data values $\left\{\left(x_{i+1}, y_{i+1}\right), \ldots\right.$, $\left.\left(x_{i+m}, y_{i+m}\right)\right\}$ toward the outside regions of the image up and down, respectively, where $x_{i+m}$ represents the row number and $y_{i+m}$ is the forecasting value at the position $x_{i+m}$.

(7) All the forecasting values resulting from steps (3) and (6) are added into the original matrix according to the corresponding indexes of the expanded rows and columns, which gives the extrapolation version of edge expansion of the given two-dimensional image.

3.2. Closed-End Mirror Expansion of the Extrapolation Version. After the extrapolation expansion of the SVM regression model is carried out, the closed-end mirror expansion method will be applied for the two-dimensional image. As a further expansion, it makes full use of the known information involved in the original data for the extrapolation has provided larger reliable expansion regions. So it may have a better performance for mitigating the edge effect caused by the decomposition process of BEMD.

In operating the closed-end mirror expansion of the extrapolation version resulting from the SVM regression model, the procedure can be briefly summarized as follows:

(1) At first, identify if the forecasting values in the $m$ expanded columns are local extrema or not. If it is true, the extrapolation operation driven by the SVM regression model is terminated, or otherwise, the operation continues to get $s$ data values until a local extremum is captured.

(2) Similarly, identify the forecasting values in the $n$ expanded rows. If there exist some local extrema, one or more than one, the extrapolation is terminated, or otherwise, it continues to get $n$ data values until a local extremum is captured.

(3) Finally, put "mirrors" on those positions of local extrema close to the edges determined in steps (1) and (2) and expand the extrapolation version of the image by traditional closed-end mirror expansion technique. In this way, a close sequence is formed, which will be used to realize the operation of the BEMD.

\section{Image Denoising by Using the BEMD}

4.1. Image Denoising. In the process of image acquisition, compression, conversion, and transmission, some noises may be mixed up with pure image information. Therefore, the real signal of the image may be generally regarded as a compound of the noise and pure image information, which can be expressed as

$$
g(m, n)=f(m, n)+z(m, n),
$$

where $g(m, n)$ is the real signal of the image with noises, $f(m, n)$ is the image signal, and $z(m, n)$ is the noise.

If the BEMD is operated to these two types of signals, assuming that $f(m, n)$ has the same numbers of IMFs as $z(m, n)$, one should have

$$
\begin{aligned}
f(m, n)= & \sum_{i=1}^{k} f_{i}(m, n)+r_{f}(m, n), \\
z(m, n)= & \sum_{i=1}^{k} z_{i}(m, n)+r_{z}(m, n), \\
g(m, n)= & \sum_{i=1}^{k} f_{i}(m, n)+r_{f}(m, n)+\sum_{i=1}^{k} z_{i}(m, n) \\
& +z_{z}(m, n),
\end{aligned}
$$

where $f_{i}(m, n)$ and $z_{i}(m, n)$ are the IMF components of $f(m, n)$ and $z(m, n)$ and $r_{f}(m, n)$ and $r_{z}(m, n)$ are their residuals. Image denoising means that the signal content $z(m, n)$ is removed from $g(m, n)$. The residual $r_{z}(m, n)$ resulting from the BEMD of $z(m, n)$ can be neglected for it is generally very small. Most importantly, after removing all $z_{i}(m, n)$, the basic characteristic information still remains in the obtained image.

The noisy image can be decomposed into some IMFs by the BEMD. These IMFs contain high frequency components in space involved in the original pure image and also include contributions from the IMFs of the noise signal. Accordingly, it is a key problem for the image denoising to remove the latter from the decomposed results of the image. It is known that the BEMD algorithm can adaptively break the original image signal into finite components on different intrinsic scales. That is to say, it should be of benefit to the extraction of information details of the image, to lay a good foundation for the following denoising processing. In general, the noise has 


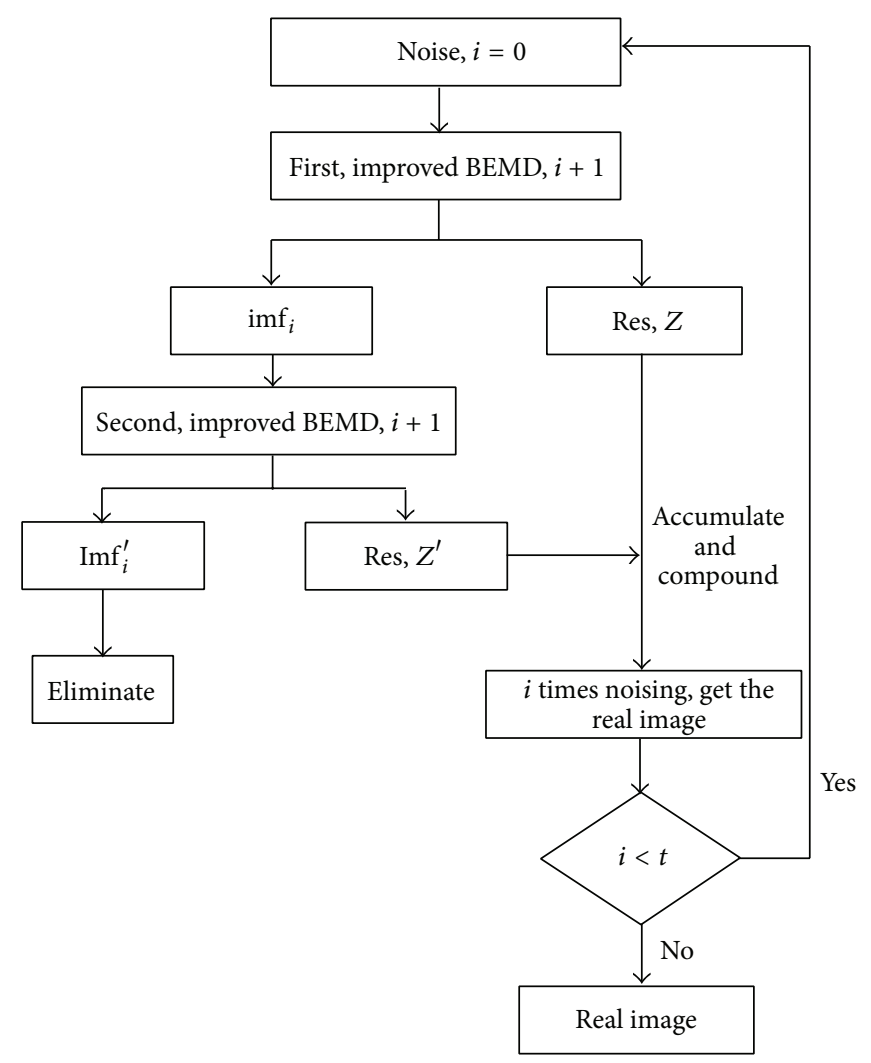

FIGURE 1: Flowchart of image denoising by the modified BEMD algorithm.

major contributions to some IMFs, but quite few to the others. Thus, only by removing those IMFs full of the noise contents can we reconstruct the real image from the original noisy one by synthesizing the remaining IMFs.

\subsection{Image Denoising Procedure by the Decomposition of} $B E M D$. In order to obtain reliable decomposition results of the BEMD, the treatment of edge effects is introduced. To some extent, it provides a modified version of the BEMD with edge treatment for enhancing the image denoising performance. The flowchart of its image denoising may be schemed as shown in Figure 1. Details of the procedure are given as follows:

(1) Firstly, input the image signal waiting for processing, and set $i=0$.

(2) Expand each edge of the image by means of the proposed approach to get the expansion signal. After that, decompose this signal by the BEMD, which is referred to as the first decomposition. In this decomposition, the noisy image signal is just broken down into one component of IMF and a residual $Z$. This IMF is regarded as the component full of noise and edge characteristics involved in the original image. At this time, the parameter $i$ is replaced with $i+1$.

(3) Further decompose the obtained IMF by the modified version of the BEMD, which is referred to as the second decomposition. In this decomposition, one can extract the residual, designated as $Z^{\prime}$.

(4) Synthesize two residual components resulting from above two decomposition operations and obtain the reconstruction image $Y=Z+Z^{\prime}$. This image is the denoised result after the $i$ th denoising. If $i<t$ ( $t$ usually takes 2 ), take $Y$ as a new original image and return to step (2), or otherwise terminate the denoising calculation process.

\section{Applications}

5.1. Image with Gaussian White Noise. Consider a $(256 \times 256-$ pixel) gray Lean image given in Figure 2(a). A Gaussian white noise is added into it to form a contaminated image by the white noise, as shown in Figure 2(b), where the Gaussian white noise has a zero mean and a variance of 0.01 . This noisy image will be used as the first example to illustrate the effectiveness of the proposed method in image denoising.

The image with Gaussian white noise, as shown in Figure 2(b), may be decomposed by the BEMD coupled with the proposed approach to improve the edge effect into a number of IMFs. With consideration of the fact that the noise signal has a characteristic of high frequency (in space), one can find that it should be mainly involved in the high frequency component decomposed by the BEMD. In other words, the denoising is essentially equivalent to an operation to remove some signal contents from the highest components 


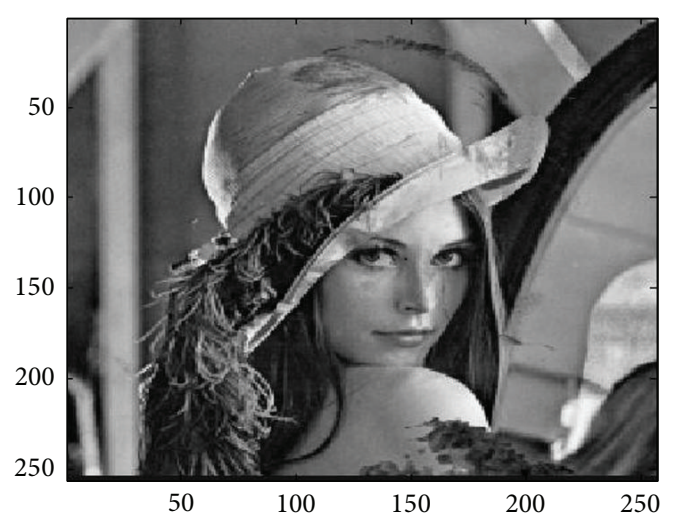

(a) Original image

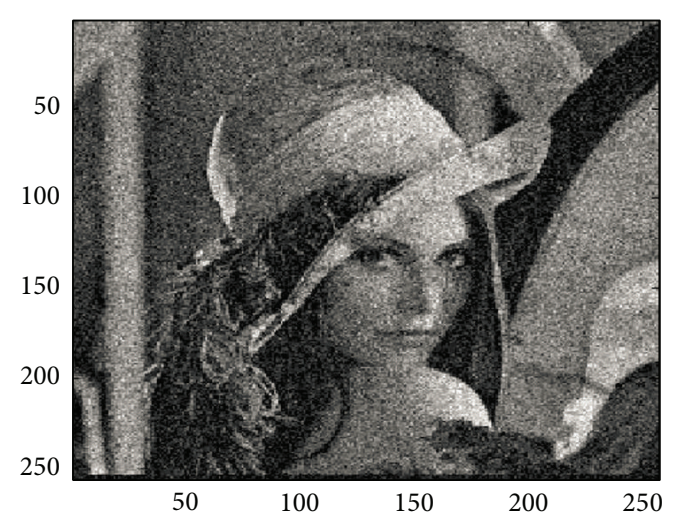

(b) Noisy image

FIGURE 2: Gray Lean image and its contaminated version by Gaussian white noise.

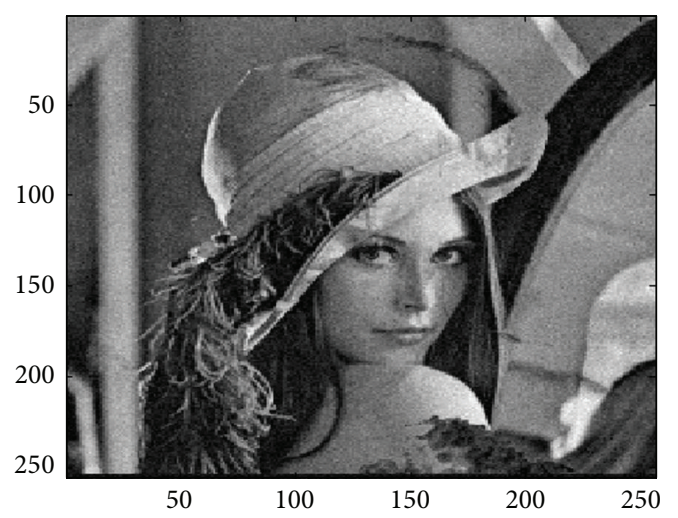

(a) The proposed approach

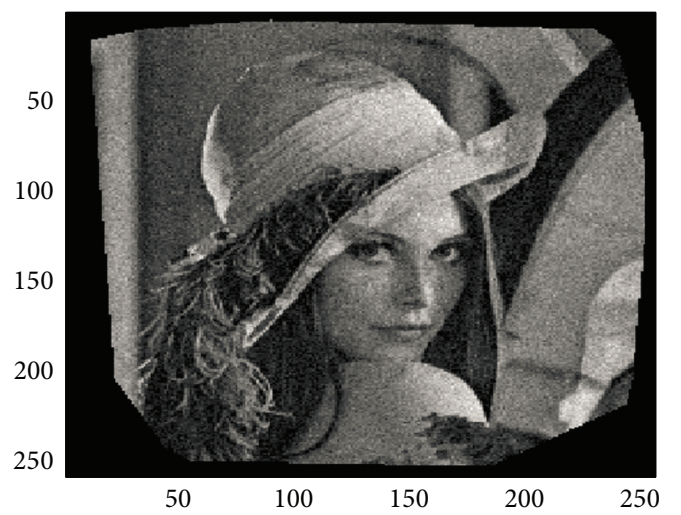

(c) Free of edge treatment

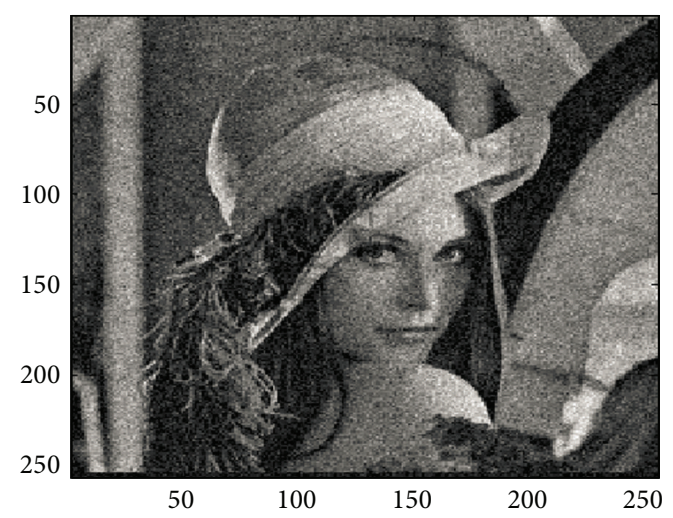

(b) Wiener method

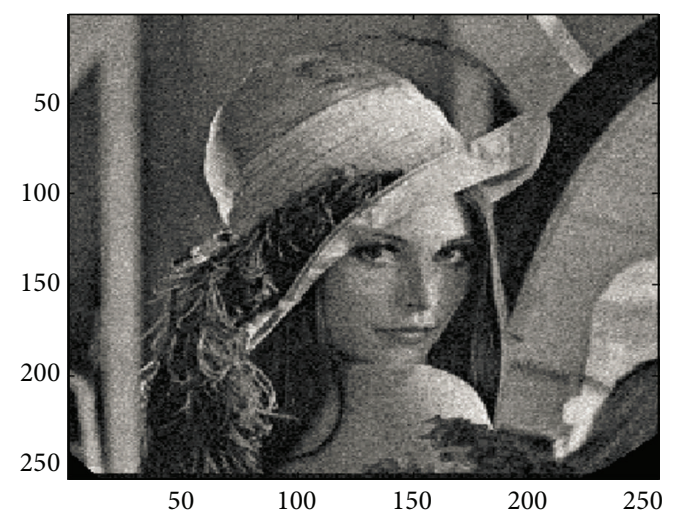

(d) Edge treatment by mirror expansion

FIGURE 3: Comparison of denoising results for the image with Gaussian white noise.

of the decomposed results. Therefore, as mentioned above in the image denoising procedure by the decomposition of BEMD algorithm, the first decomposition of the noisy image signal is done for only one time to extract an IMF, and then this IMF, as a new signal, completes another decomposition, obtaining another IMF. The IMF resulting from the latter may be approximately regarded as the noise. Subtracting it from the original noisy signal yields a new image, which is the result of the image denoising, as shown in Figure 3(a).
Compared with the result denoised by the Wiener method, as shown in Figure 3(b), it can be seen that the proposed method has a better performance. On the other hand, if the edge effect is not considered and the BEMD is used to denoise the image through the same procedure as the proposed method, one can obtain the result of the image denoising free of edge treatment, as shown in Figure 3(c). Obviously, the edge effect has serious influence on the result of the denoising by the BEMD. When the edge effect is treated 


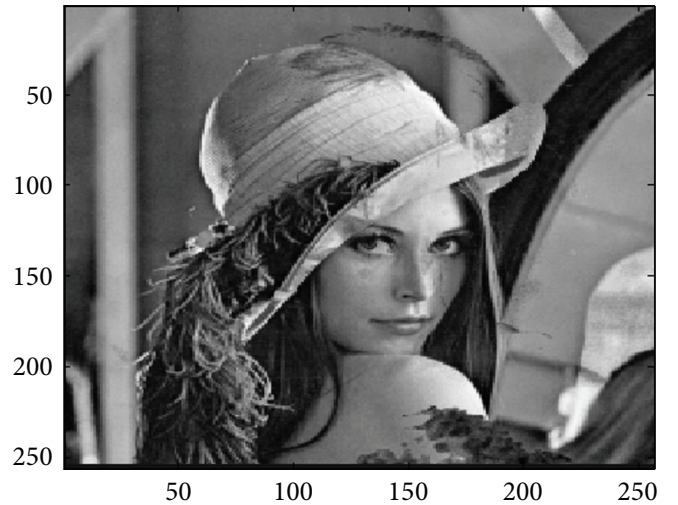

(a) Original image

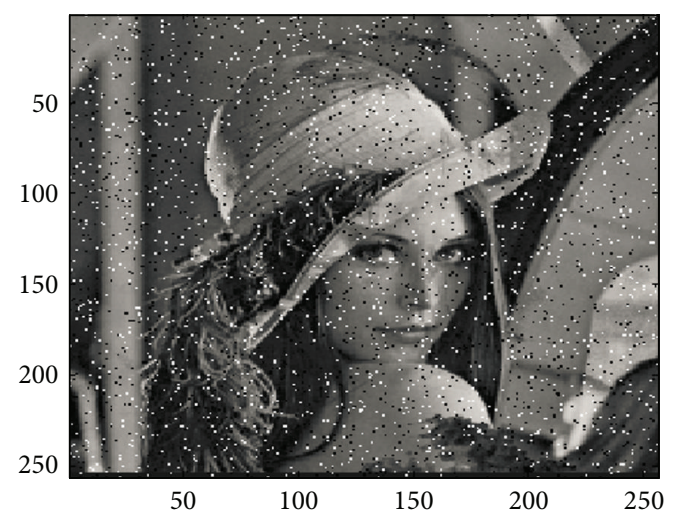

(b) Noisy image

FIGURE 4: Gray Lean image and its contaminated version by salt and pepper noise.

by the mirror expansion approach in the decomposition of the BEMD, the denoised image is shown in Figure 3(d). This result is better than that by the Wiener method. It is also better than the case without consideration of the edge effect but worse than that obtained by the proposed method, which shows that it is very important for image denoising by the BEMD to improve the edge effect, and also illustrates a good denoising result of the BEMD coupled with the proposed approach.

5.2. Image with Salt and Pepper Noise. When the salt and pepper noise is added to the gray Lean image in Figure 2(a) (for convenience of comparison, it is presented again in Figure 4(a)), an image with salt and pepper noise is obtained, as shown in Figure 4(b). This noisy image is the second example to illustrate the effectiveness of the proposed method in image denoising. The added salt and pepper noise has a density of 0.05 .

Similarly to the first example of the denoising of the image with Gaussian white noise, with regard to the noisy image in Figure 4(b), a denoising process can be done by following the procedure described in Figure 1. The results include ones obtained by the proposed approach, Wiener method, BEMD free of edge treatment, and BEMD with edge treatment by mirror expansion, as shown in Figures 5(a), 5(b), 5(c), and 5(d), respectively. It can be seen that the proposed approach gives a more satisfactory result than the others. The BEMD free of edge treatment does not recover the picture frame for the influence of the edge effect. But this default can be remedied by the BEMD with edge treatment of mirror expansion. Compared with the proposed approach, it leads to a lower life-like appearance of the image. Because of this, any spots contaminated by the noise almost cannot be seen from the denoised image. However, some of these spots still remain in the result from the proposed approach. It shows that some contents of the noise enter the other IMFs except for the first IMF. Among them, the Wiener method has a poorest performance for treating the salt and pepper noise.
5.3. Image with Random Noise. The original gray Lean image is presented in Figure 6(a). If adding 20\% random noise to it, we can get a noisy image, as shown in Figure 6(b).

As expected, the proposed approach gives the best result of removing the random noise; see Figure $7(\mathrm{a})$. Both Wiener method and the BEMD with edge treatment by mirror expansion may reach an acceptable result of the denoising, as shown in Figures 7(b) and 7(c). However, compared with the denoised image by the proposed approach, those from both of them still maintain a definite distance. It shows that the schedule combining the extrapolation expansion with the closed-end mirror expansion really provides an effective approach for improving the edge effect of the BEMD operation to get the reliable decomposed results and then enhance the image denoising performance of the BEMD by a big margin.

5.4. Estimation of Denoising Effect. To quantitatively interpret the effectiveness of the proposed denoising method, let us have a look at the peak signal-to-noise ratio (PSNR). The PSNR is an expression for the ratio between the maximum possible power of a signal and the power of corrupting noise that affects the fidelity of its representation, and it is most commonly used to measure the effect of image enhancement algorithm on image quality [42]. Because many signals have a very wide dynamic range, PSNR is usually expressed in terms of the logarithmic decibel scale and defined as [42]

$$
\text { PSNR }=10 \log _{10}\left(\frac{R^{2}}{\mathrm{MSE}}\right),
$$

where $R$ is the maximum possible pixel value of the image and MSE is the mean squared error, given by

$$
\text { MSE }=\frac{1}{m \times n} \sum_{x=1}^{m} \sum_{y=1}^{n}\left[f(x, y)-f^{\prime}(x, y)\right] .
$$

In (19), $f(x, y)$ is the matrix data of original image without noise, $f^{\prime}(x, y)$ is the matrix data of denoised image, and $m \times n$ represents the matrix size of the image under consideration. 


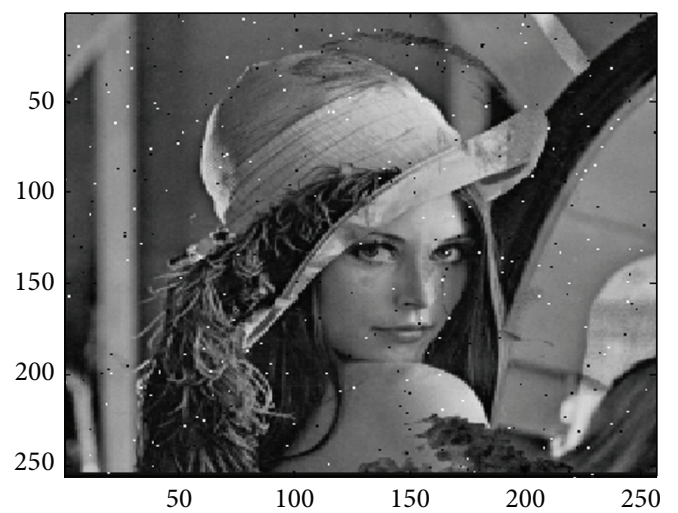

(a) The proposed approach

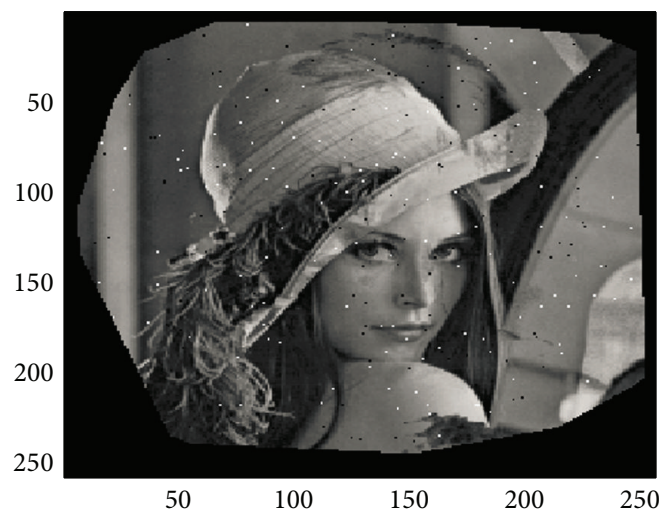

(c) Free of edge treatment

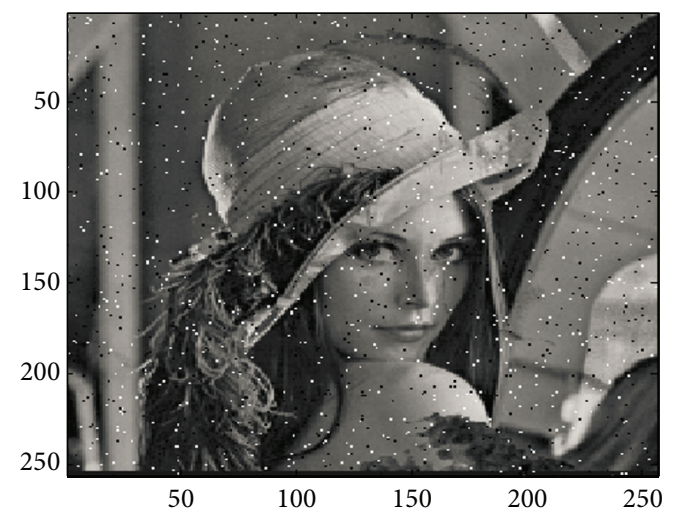

(b) Wiener method

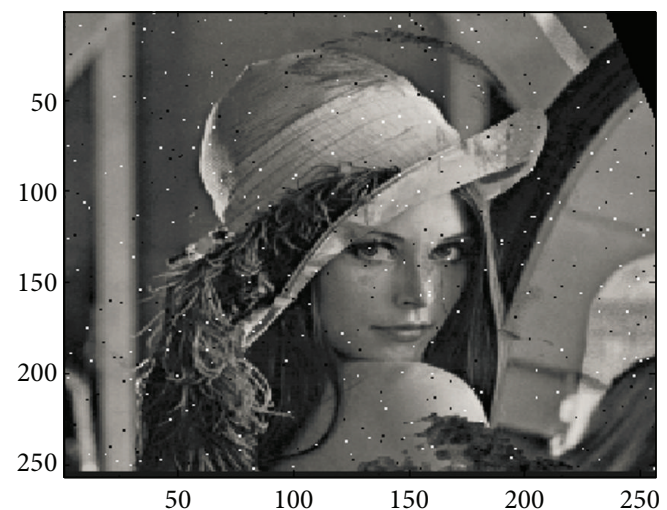

(d) Edge treatment by mirror expansion

FIGURE 5: Comparison of denoising results for the image with salt and pepper noise.

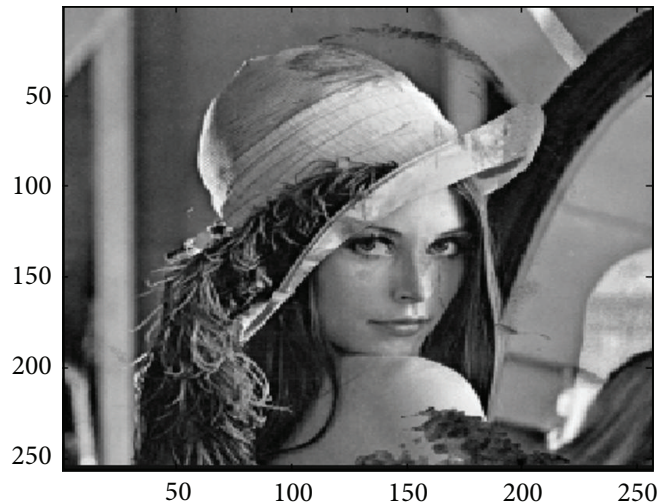

(a) Original image

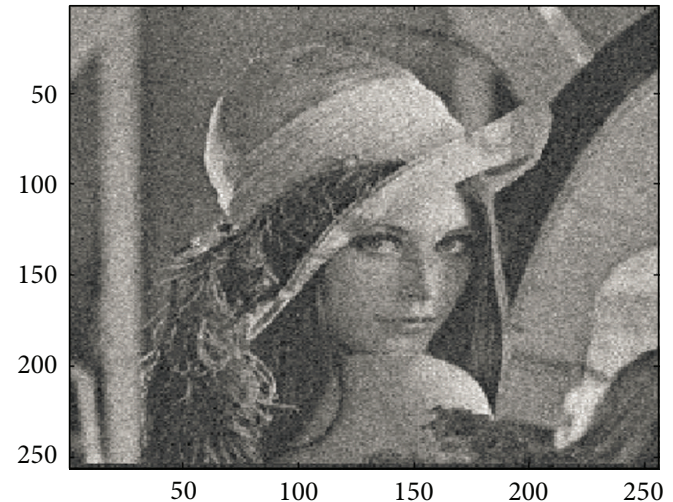

(b) Noisy image

FIGURE 6: Gray Lean image and its contaminated version by $20 \%$ random noise.

According to the definition of PSNR given by (18), we calculate the value for each image before and after denoising. All results are listed, as shown in Table 1. Compared with the PSNRs before denoising, the values after denoising increase to some extent for any one of the above four denoising methods. The proposed approach gets a first in increasing the PSNR, and the next one is the BEMD free of edge treatment. Relatively, the Wiener method plays a worst role in improving the PSNR after denoising. Although the BEMD free of edge treatment results in higher values of PSNR than the BEMD with edge treatment by mirror expansion, it cannot recover the whole picture after denoising, which can 
TABLE 1: Peak signal to noise ratios (PSNRs) of the noisy and denoising images.

\begin{tabular}{|c|c|c|c|c|c|c|c|c|c|c|}
\hline \multirow[t]{2}{*}{ Noise type } & \multicolumn{2}{|c|}{ Before denoising } & \multicolumn{2}{|c|}{ Wiener method } & \multicolumn{2}{|c|}{ The proposed approach } & \multicolumn{2}{|c|}{ Free of edge treatment } & \multicolumn{2}{|c|}{$\begin{array}{l}\text { Edge treatment by } \\
\text { mirror expansion }\end{array}$} \\
\hline & MSE & PSNR & MSE & PSNR & MSE & PSNR & MSE & PSNR & MSE & PSNR \\
\hline Gaussian white noise & 616.07 & 20.23 & 373.65 & 22.41 & 191.32 & 25.31 & 204.40 & 24.03 & 256.88 & 23.03 \\
\hline Salt and pepper noise & 975.80 & 18.24 & 398.16 & 22.13 & 62.49 & 30.17 & 99.96 & 28.13 & 137.25 & 26.76 \\
\hline $20 \%$ random noise & 400.43 & 22.11 & 100.95 & 28.09 & 3.97 & 42.15 & 15.98 & 36.09 & 16.81 & 35.88 \\
\hline
\end{tabular}

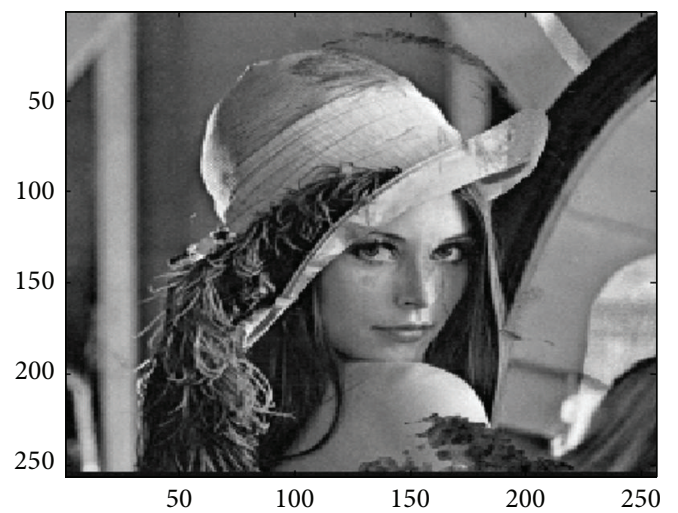

(a) The proposed approach

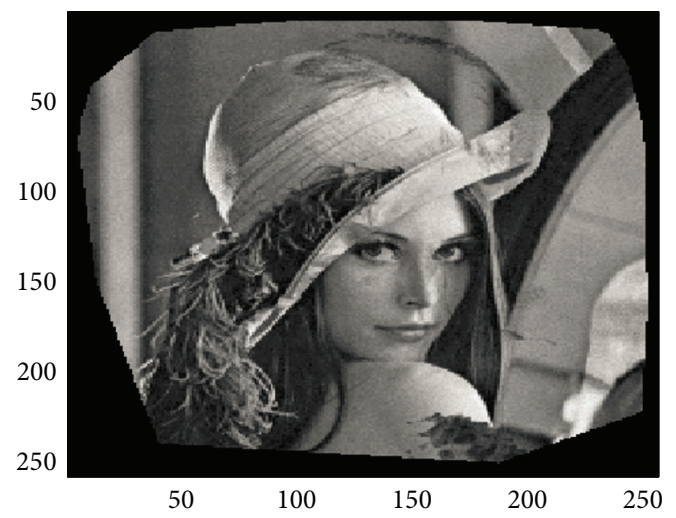

(c) Free of edge treatment

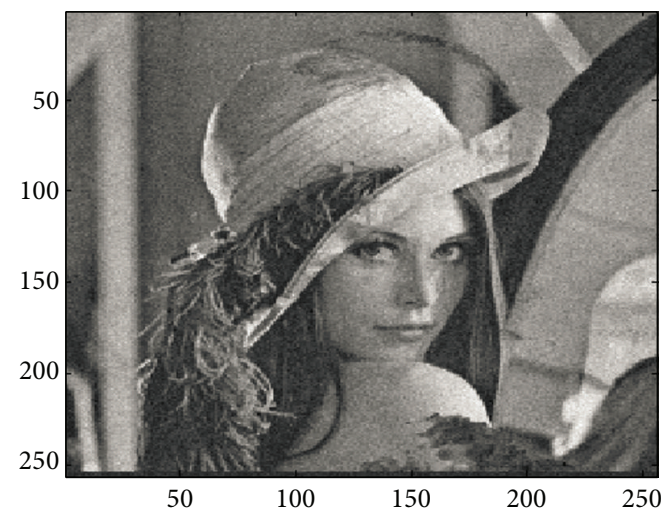

(b) Wiener method

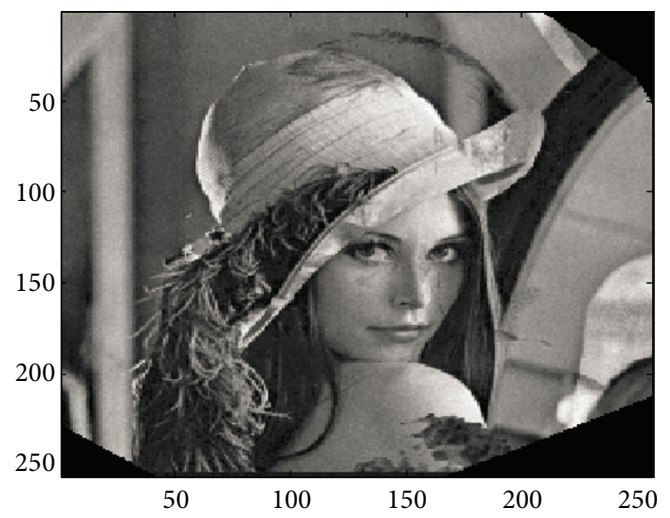

(d) Edge treatment by mirror expansion

FIgURE 7: Comparison of denoising results for the image with $20 \%$ random noise.

be seen clearly in Figures 3(c), 5(c), and 7(c). Consequently, its denoising effect is not really better than the BEMD with edge treatment by mirror expansion. In Table 1, all the MSEs related to PSNRs are simultaneously presented. Since they represent the mean squared errors, the same conclusion can be drawn out from them as the PSNRs, which shows that the most satisfactory denoising effect can be reached by the decomposition of BEMD with edge treatment of the SVM extrapolation coupled with the closed-end mirror expansion.

\section{Conclusions}

Image denoising by the BEMD is an interesting concept, introduced about ten years ago, where filtering is accomplished through the decomposed results on different intrinsic scales instead of the image itself. However, to enhance the
BEMD performance for noise removal and other image processing applications, the edge effect is an essential problem worthy of notice, in addition to the development of fast and more effective methods. This fact motivated the formulation of the proposed approach to improving the edge effect to enhance the denoising performance of the BEMD. The edge treatment includes two steps, in which the first one is an extrapolation operation through the model constructed by the SVM based on the information of the original signal and the second is an expansion by the closed-end mirror expansion technique with respect to the extrema nearest to and beyond the edge of the data resulting from the first operation.

With consideration of the fact that the highest noise content of an image is extracted into the first IMF and the remaining fewer noise contents are extracted into subsequent IMFs, this work introduces a procedure of the image 
denoising through the modified version of the BEMD. The first decomposition just breaks down the noisy image into one component of IMF and a residual, and the second decomposition extracts a residual from the IMF of the first decomposition. Synthesizing these two residual components obtains the reconstruction image, which is the denoised result.

Applications to the gray Lean images contaminated by Gaussian white noise, salt and pepper noise, and random noise show that the proposed approach has an amazing ability for enhancing the image denoising performance of the BEMD. It not only overcomes some drawbacks in the BEMD free of edge treatment, but also improves the performance of the BEMD with edge treatment by mirror expansion. Compared with the Wiener method, the BEMD coupled with edge treatment of the proposed approach shows better results for the given three types of noises, in a significant reduction of noise without loss of desired image contents. Further, the PSNRs of the denoised images confirm the best performance of the BEMD by improving the edge effect.

\section{Conflict of Interests}

The authors declare that there is no conflict of interests regarding the publication of this paper.

\section{Acknowledgment}

This work is supported by the Fundamental Research Funds for the Central Universities of China.

\section{References}

[1] U. Diewald, S. Morigi, and M. Rumpf, "A cascadic geometric filtering approach to subdivision," Computer Aided Geometric Design, vol. 19, no. 9, pp. 675-694, 2002.

[2] J.-L. Starck, E. J. Candès, and D. L. Donoho, "The curvelet transform for image denoising," IEEE Transactions on Image Processing, vol. 11, no. 6, pp. 670-684, 2002.

[3] R. Yan, L. Shao, and Y. Liu, "Nonlocal hierarchical dictionary learning using wavelets for image denoising," IEEE Transactions on Image Processing, vol. 22, no. 12, pp. 4689-4698, 2013.

[4] B. K. S. Kumar, "Image denoising based on non-local means filter and its method noise thresholding," Signal, Image and Video Processing, vol. 7, no. 6, pp. 1211-1227, 2013.

[5] J. H. Kim and K. N. Choi, "Range image denoising using a constrained local Gaussian model for 3D object query service in the smart space," Personal and Ubiquitous Computing, vol. 17, no. 7, pp. 1401-1407, 2013.

[6] F. Adamo, G. Andria, F. Attivissimo, A. M. L. Lanzolla, and M. Spadavecchia, "A comparative study on mother wavelet selection in ultrasound image denoising," Measurement, vol. 46, no. 8, pp. 2447-2456, 2013.

[7] J. Portilla, V. Strela, M. J. Wainwright, and E. P. Simoncelli, "Image denoising using scale mixtures of Gaussians in the wavelet domain," IEEE Transactions on Image Processing, vol. 12, no. 11, pp. 1338-1351, 2003.

[8] X.-Y. Wang and Z.-K. Fu, "A wavelet-based image denoising using least squares support vector machine," Engineering Applications of Artificial Intelligence, vol. 23, no. 6, pp. 862-871, 2010.
[9] D. Zhang, S. Mabu, and K. Hirasawa, "Image denoising using pulse coupled neural network with an adaptive Pareto genetic algorithm," IEEJ Transactions on Electrical and Electronic Engineering, vol. 6, no. 5, pp. 474-482, 2011.

[10] X.-D. Gu, C.-Q. Cheng, and D.-H. Yu, "Noise-reducing of four-level image using PCNN and fuzzy algorithm," Journal of Electronics and Information Technology, vol. 25, no. 12, pp. 15851598, 2003.

[11] Y. Yan and B. Guo, "Two image denoising approaches based on wavelet neural network and particle swarm optimization," Chinese Optics Letters, vol. 5, no. 2, pp. 82-85, 2007.

[12] J. Zhang, Z. Lu, L. Shi, J. Dong, and M. Shi, "Filtering images contaminated with pep and salt type noise with pulse-coupled neural networks," Science in China, Series F: Information Sciences, vol. 48, no. 3, pp. 322-334, 2005.

[13] L. Dehyadegary, S. A. Seyyedsalehi, and I. Nejadgholi, "Nonlinear enhancement of noisy speech, using continuous attractor dynamics formed in recurrent neural networks," Neurocomputing, vol. 74, no. 17, pp. 2716-2724, 2011.

[14] X.-Y. Wang, H.-Y. Yang, Y. Zhang, and Z.-K. Fu, "Image denoising using SVM classification in nonsubsampled contourlet transform domain," Information Sciences, vol. 246, pp. 155-176, 2013.

[15] N. E. Huang, Z. Shen, S. R. Long et al., "The empirical mode decomposition and the Hilbert spectrum for nonlinear and non-stationary time series analysis," Proceeding of Royal Society London: A, vol. 454, no. 12, pp. 903-995, 1998.

[16] J.-Y. Huang, K.-L. Wen, X.-J. Li, J.-J. Xie, C.-T. Chen, and S.C. $\mathrm{Su}$, "Coseismic deformation time history calculated from acceleration records using an EMD-derived baseline correction scheme: a new approach validated for the 2011 Tohoku earthquake," Bulletin of the Seismological Society of America, vol. 103, no. 2, pp. 1321-1335, 2013.

[17] A. Garcia-Perez, J. P. Amezquita-Sanchez, A. DominguezGonzalez, R. Sedaghati, R. Osornio-Rios, and R. J. RomeroTroncoso, "Fused empirical mode decomposition and wavelets for locating combined damage in a truss-type structure through vibration analysis," Journal of Zhejiang University: Science A, vol. 14, no. 9, pp. 615-630, 2013.

[18] W. Huang, Z. Shen, N. E. Huang, and Y. C. Fung, "Use of intrinsic modes in biology: examples of indicial response of pulmonary blood pressure to \pm step hypoxia," Proceedings of the National Academy of Sciences of the United States of America, vol. 95, no. 22, pp. 12766-12771, 1998.

[19] J. Zheng, J. Cheng, and Y. Yang, "Generalized empirical mode decomposition and its applications to rolling element bearing fault diagnosis," Mechanical Systems and Signal Processing, vol. 40, no. 1, pp. 136-153, 2013.

[20] K. Zhu, X. Song, and D. Xue, "Incipient fault diagnosis of roller bearings using empirical mode decomposition and correlation coefficient," Journal of Vibroengineering, vol. 15, no. 2, pp. 597603, 2013.

[21] H. Song, Y. Bai, L. Pinheiro, C. Dong, X. Huang, and B. Liu, "Analysis of ocean internal waves imaged by multichannel reflection seismics, using ensemble empirical mode decomposition," Journal of Geophysics and Engineering, vol. 9, no. 3, pp. 302-311, 2012.

[22] J. C. Nunes, Y. Bouaoune, E. Delechelle, O. Niang, and P. Bunel, "Image analysis by bidimensional empirical mode decomposition," Image and Vision Computing, vol. 21, no. 12, pp. 1019-1026, 2003. 
[23] J. C. Nunes, S. Guyot, and E. Deléchelle, "Texture analysis based on local analysis of the bidimensional empirical mode decomposition," Machine Vision and Applications, vol. 16, no. 3, pp. 177-188, 2005.

[24] A. Linderhed, "2D empirical mode decompositions in the spirit of image compression," in Wavelet and Independent Component Analysis Applications IX, vol. 4738 of Proceedings of SPIE, pp. 1-8, March 2002.

[25] A. Linderhed, "Compression by image empirical mode decomposition," in Proceedings of the IEEE International Conference on Image Processing (ICIP '05), pp. 553-556, Genoa, Italy, September 2005.

[26] Z. He, Q. Wang, Y. Shen, J. Jin, and Y. Wang, "Multivariate gray model-based bemd for hyperspectral image classification," IEEE Transactions on Instrumentation and Measurement, vol. 62, no. 5, pp. 889-904, 2013.

[27] Y. Zhou and H. Li, "Adaptive noise reduction method for DSPI fringes based on bi-dimensional ensemble empirical mode decomposition," Optics Express, vol. 19, no. 19, pp. 18207-18215, 2011.

[28] L. Qiao, K. Niu, N. Wang, and L. Peng, "Perfect reconstruction image modulation based on BEMD and quaternionic analytic signals," Science China-Information Sciences, vol. 54, no. 12, pp. 2602-2614, 2011.

[29] Y. Chen, L. Wang, Z. Sun, Y. Jiang, and G. Zhai, "Fusion of color microscopic images based on bidimensional empirical mode decomposition," Optics Express, vol. 18, no. 21, pp. 21757-21769, 2010.

[30] A. Linderhed, Adaptive image compression with wavelet paekets and empirical mode decomposition [Ph.D. thesis], Linköping Studies in Science and Technology, 2004.

[31] Z.X. Liu and S. L. Peng, "Boundary processing of bidimensional EMD using texture synthesis," IEEE Signal Processing Letters, vol. 12, no. 1, pp. 33-36, 2005.

[32] V. T. Tran, B.-S. Yang, F. Gu, and A. Ball, “Thermal image enhancement using bi-dimensional empirical mode decomposition in combination with relevance vector machine for rotating machinery fault diagnosis," Mechanical Systems and Signal Processing, vol. 38, no. 2, pp. 601-614, 2013.

[33] Y. Ye, "Adaptive boundary effect processing for empirical mode decomposition using template matching," Applied Mathematics \& Information Sciences, vol. 7, no. 1, pp. 61-66, 2013.

[34] Y. Deng, W. Wang, C. Qian et al., "The EMD method and deal with the boundary problem in the Hilbert transform," Chinese Science Bulletin, vol. 46, no. 3, pp. 257-263, 2001.

[35] W. Xiaofeng and P. Hao, "Study of HHT end effect suppression based on RBF extension," International Journal of Advancements in Computing Technology, vol. 4, no. 13, pp. 369-377, 2012.

[36] T. Xiong, Y. Bao, and Z. Hu, "Does restraining end effect matter in EMD-based modeling framework for time series prediction? Some experimental evidences," Neurocomputing, vol. 123, pp. 174-184, 2014.

[37] Z. He, Y. Shen, Q. Wang, Y. Wang, N. Feng, and L. Ma, "Mitigating end effects of EMD using non-equidistance grey model," Journal of Systems Engineering and Electronics, vol. 23, no. 4, pp. 603-611, 2012.

[38] W.-P. Hu, J.-L. Mo, Y.-J. Gong, F.-W. Zhao, and M.-H. Du, "Methods for mitigation of end effect in empirical mode decomposition: a quantitative comparison," Dianzi Yu Xinxi Xuebao, vol. 29, no. 6, pp. 1394-1398, 2007.
[39] J. L. Sanchez and J. J. Trujillo, "Improving the empirical mode decomposition method," Applicable Analysis, vol. 90, no. 3-4, pp. 689-713, 2011.

[40] V. N. Vapnik, The Nature Of Statistical Learning Theory, Springer-Verlag, New York, NY, USA, 1995.

[41] V. Vapnik and A. Vashist, "A new learning paradigm: learning using privileged information," Neural Networks, vol. 22, no. 5, pp. 544-557, 2009.

[42] Q. Huynh-Thu and M. Ghanbari, "Scope of validity of PSNR in image/video quality assessment," Electronics Letters, vol. 44, no. 13, pp. 800-801, 2008. 

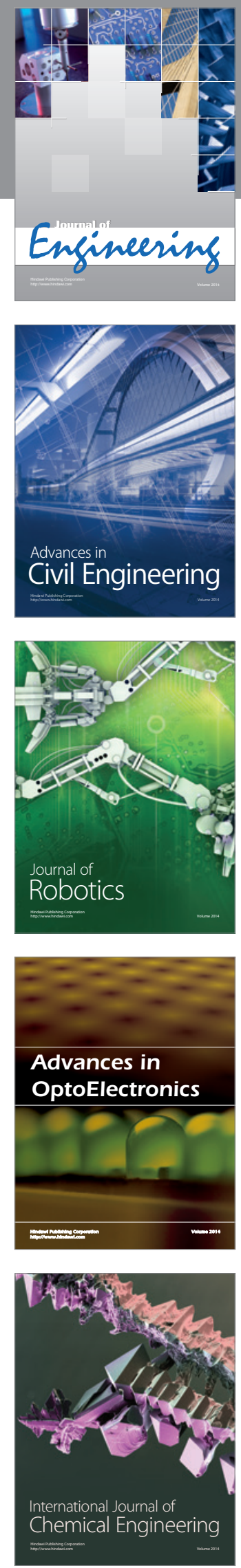

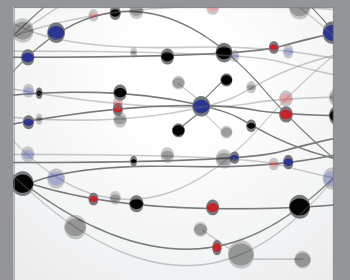

The Scientific World Journal
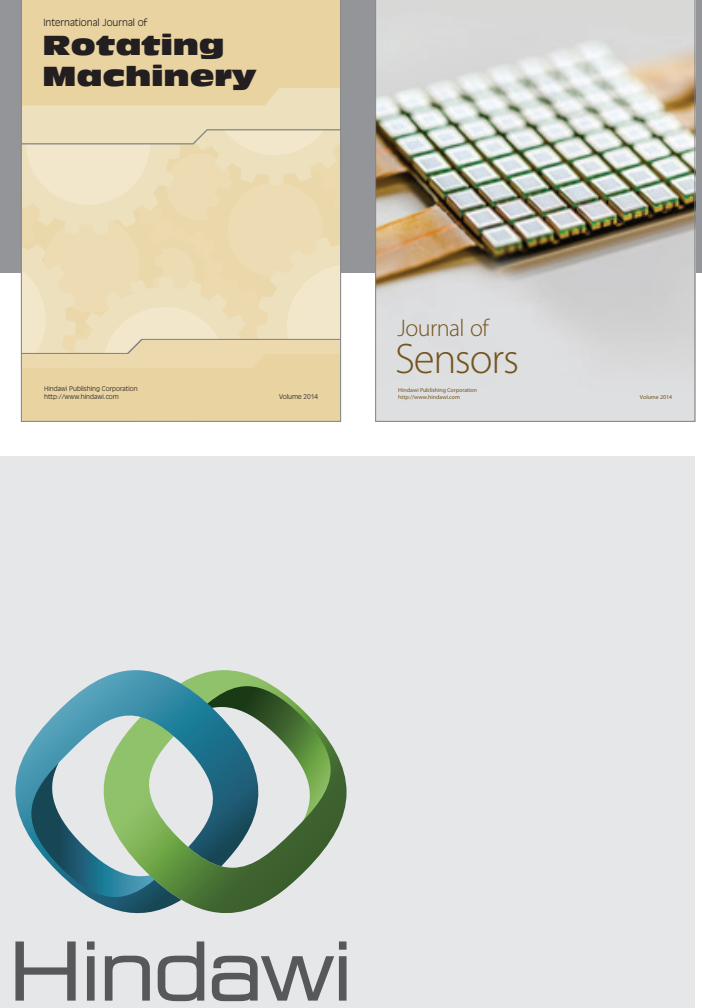

Submit your manuscripts at http://www.hindawi.com
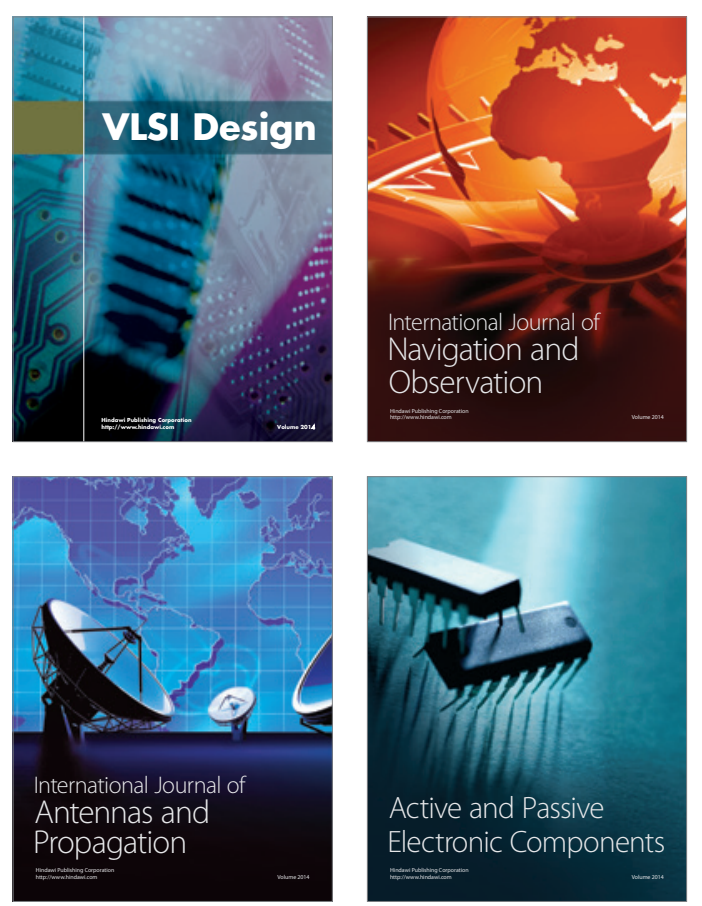
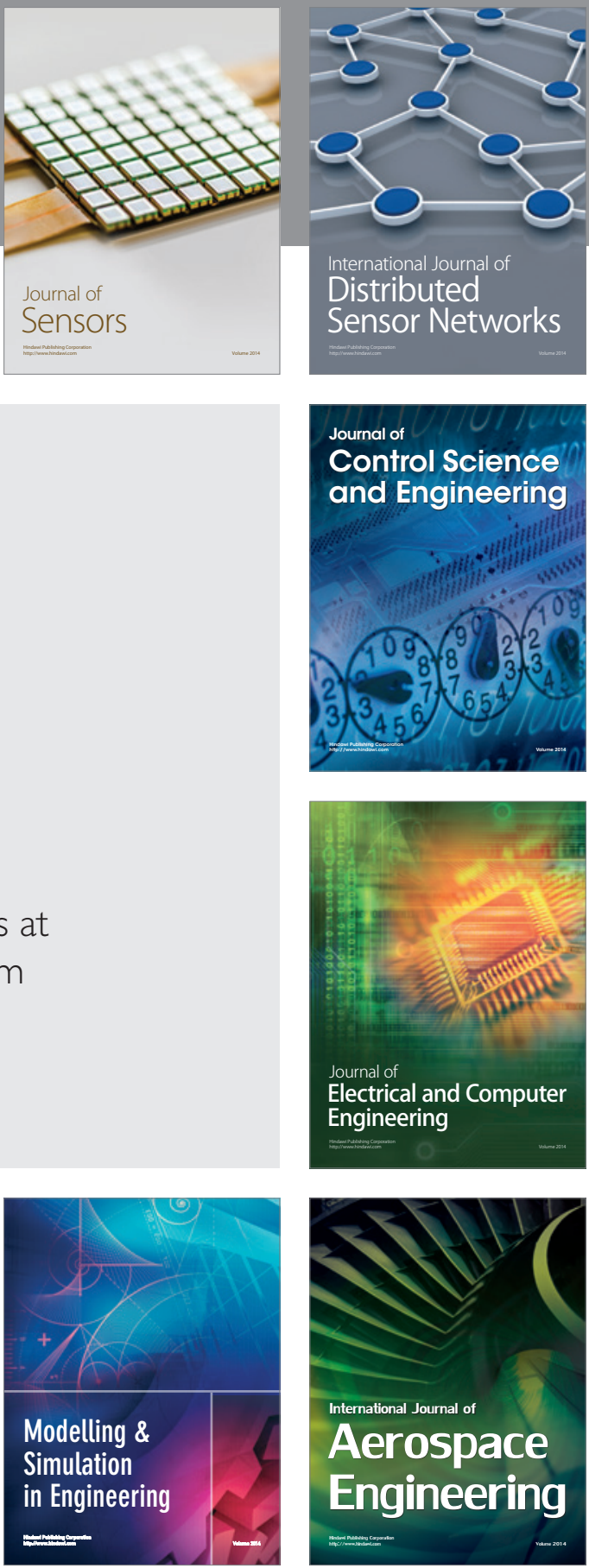

Journal of

Control Science

and Engineering
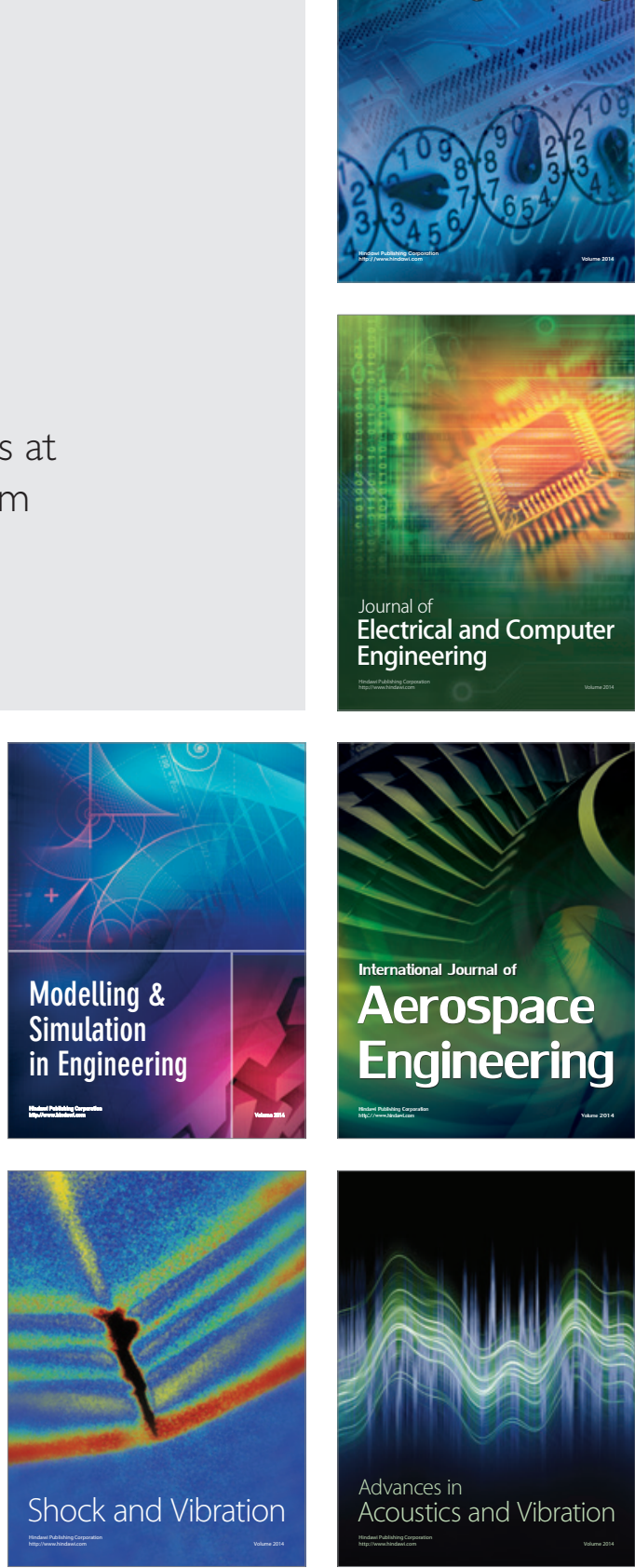